\title{
Pesquisas
}

\section{Ucrania, Rusia y los flujos energéticos}

Carlos A. García López*

Luego que iniciaran las protestas de parte del pueblo ucraniano ante la negativa de sus autoridades de firmar un acuerdo propuesto por la Unión Europea retomó importancia la dependencia energética que abriga esta región, toda vez que gran parte del gas natural ruso que consume debe viajar por Ucrania, quien ha enfrentado diversos desacuerdos con Gazprom que culminaron en recortes al flujo de gas afectando el abastecimiento de la UE. Además los continuos avatares económicos y políticos siguen afectando a Ucrania que se presenta como un caso particular poco estudiado y atendido. Con la finalidad de profundizar en lo antes mencionado el presente documento se ha dividido en dos apartados: el primero señala algunos sucesos económicos y sociales por los que atravesó Ucrania luego de su independencia para después hacer un recuento breve de las causas del conflicto en que se sumerge actualmente esta nación; en el segundo se abordan los aspectos energéticos de la UE y algunas implicaciones derivadas de la posición que ocupa Ucrania como paso obligado de parte del gas natural que Rusia vende al bloque europeo. Finalmente se presentan algunas reflexiones a modo de conclusiones.

\section{Desempeño general} de Ucrania

A raíz de la obtención de su soberanía en 1991 Ucrania vivió -y continúa experimentando- numerosas y muy intensas adversidades. En sus primeros años de vida independiente esta nación se vió afectada por continuas variaciones negativas - por debajo de las cero unidades- en su producción nacional, que llegaron a su máximo desplome en 1994 cuando el PIB se contrajo casi 23 puntos, haciendo a este país un $20 \%$ más pobre. En una economía abierta una caída de esta magnitud inevitablemente dispara la deuda pública, porque la emisión de bonos crece y cada vez a una tasa de interés más elevada -por el riesgo de defaul en el que incurre la economía. Entonces, si en Ucrania la producción real se estaba contrayendo en tal magnitud y suponemos que la inflación es función de la emisión monetaria, podemos sugerir que esta nación comenzó a imprimir dinero con el fin de licuar deuda, es decir, monetizó el déficit, acción que naturalmente hizo que la inflación se disparará alcanzando su valor máximo en 1993, cuando el nivel de precios creció a una tasa 
mensual aproximada de $38.15 \%{ }^{1}$ sumergiendo a Ucrania en una de las peores crisis (véase Gráfica 1$){ }^{2}$
Y aunque a partir del año 2000 Ucrania comenzó a presentar datos positivos en su producto nacional, in-

\section{Gráfica I}

Ucrania: PIB e inflación, 1991-1996

-variación porcentual-

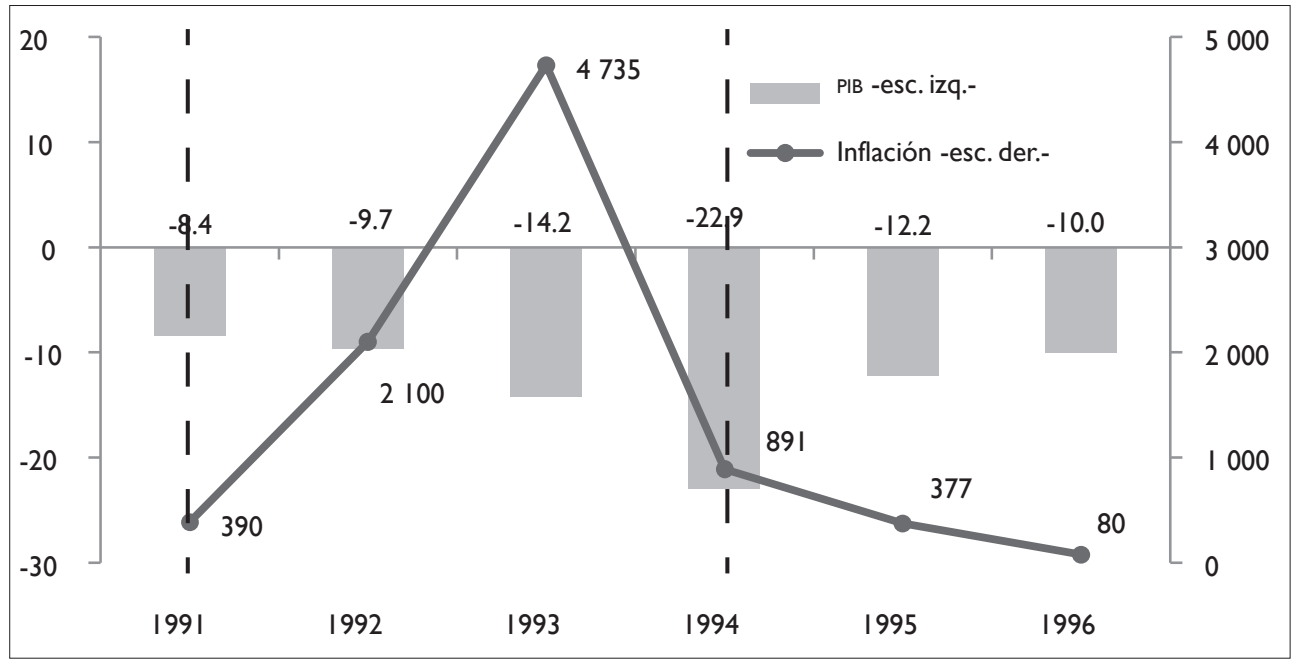

Nota: la línea vertical de 1991 muestra el año en que Ucrania obtuvo su independencia y la línea de 1994 señala el período en que comenzaron las reformas económicas.

Fuente: elaboración propia con base en datos de National Bank of Ukraine, 2015 y Banco Mundial, 2015.

${ }^{1}$ Determinada como sigue:

$$
\left[(1+r)^{n}-1\right]=r_{T},
$$

donde $r_{T}$ es el valor de la tasa acumulada al final del periodo, $r$ la tasa de cada periodo y $n$ el número de periodos obteniendo así mediante aproximación una tasa de inflación mensual del $38.15 \%$ cómo se ve a continuación:

$$
\left[(1+0.3815)^{12}-1\right] \approx 4,735
$$

${ }^{2}$ En México durante la crisis de 1994 se presentó una contracción del PIB de apenas un 5.8\% y el país vivía un completo caos, para rescatar la economía tuvieron que intervenir algunos organismos internacionales; en el caso Ucrania hubo caídas de más del 10\% en su producción nacional y hasta por cuatro años consecutivos y a ellos nadie los rescató. mediatamente, en 2004, un nuevo avatar político sacudió a la capital de ese país (Kiev) cuando los adeptos de Occidente y Rusia se enfrentaron en las elecciones presidenciales. En este episodio Victor Yushchenko -partidario de las reformas de mercado que se aplicaron desde 1994 y seguidor de Occidente- perdió los comicios frente a Viktor Yanukovich (candidato del Partido de las Regiones); inconforme con este resultado y denunciando diversas irregularidades en los 
sufragios, Yushchenko convocó a sus seguidores a manifestarse en las calles. Ante esta presión el Tribunal Supremo de Ucrania decidió declarar inválidas las elecciones, permitiendo una segunda vuelta que colocó a Victor Yushchenko como presidente; esta serie de eventos es lo que conocemos como la "Revolución Naranja" (Burdeina, 2014). Una vez cumplido su mandato, este presidente fue sustituido por el mismo Viktor Yanukovich, que se convirtió en el cuarto presidente en la era de Ucrania independiente después de ganar las elecciones de 2010; este personaje, como se verá, es clave en el desarrollo del conflicto al interior de este país por su intensa afinidad a los intereses rusos.

En el plano internacional Ucrania había enfrentado, en los últimos años, una disyuntiva entre dos alternativas de integración económica, una opción era el Acuerdo de Asociación con la ue propuesto desde Bruselas y la otra estaba constituida por la Unión Aduanera Euroasiática ${ }^{3}$ ofertada por Moscú; ambas incompatibles entre sí en cuanto a los intereses comerciales de cada lado. El Acuerdo planteado por la UE se negociaba desde 2007 pero Rusia ejercía continuas presiones sobre Ucrania para evitar que dicho acuerdo se concreta-

\footnotetext{
${ }^{3}$ Que es diferente de los tratados de libre comercio, ya que bajo este mecanismo los miembros establecen tarifas arancelarias comunes hacia países externos al convenio.
}

ra; un episodio de ello, entre los más recientes, fue el suscitado en agosto de 2013, cuando el Servicio Aduanero de la Federación de Rusia sometió a revisiones exhaustivas las exportaciones ucranianas, provocando el estancamiento de diversas mercancías, hecho que a todas luces respondía a los avances en las negociaciones en torno al Acuerdo de Asociación y a declaraciones como la del portavoz de Comercio de la Comisión Europea John Clancy y del primer ministro ucraniano Nikolái Azárov sobre las amplias posibilidades de concretar el Acuerdo con la UE durante la cumbre de la Asociación Oriental celebrada en Vilna, capital de Lituania, en noviembre de 2013.

Aunado a lo anterior existían amenazas más directas. En el mismo mes de agosto el Presidente de Rusia Vladimir Putin, declaró que los miembros de la Unión Aduanera -hasta 2013 constituida por Bielorrusia, Kazajistán y Rusia- tomarían medidas arancelarias contra todo producto proveniente de Ucrania si ésta concretaba el acuerdo con el bloque europeo, ya que las autoridades de estas naciones no estaban dispuestas a dar beneficios a mercancías provenientes de la UE. ${ }^{4}$ Como consecuencia de estas presiones, llegado el mes de

El argumento era que la firma del tratado inundaría el mercado ucraniano con mercancías europeas, lo que haría imposible diferenciar el origen de los productos. 
noviembre -durante la cumbre- el entonces presidente ucraniano Víktor Yanukovich rechazó firmar el Acuerdo con la UE -el mandatario pedía como condición un programa de ayuda financiera para cubrir adeudos de gas y modernizar los gasoductosanunciando a la vez un fortalecimiento de sus relaciones con Rusia, suceso que provocó el descontento de la población ucraniana proeuropea, que comenzó una serie de manifestaciones. Pese a ello un mes después, pasando por alto las protestas, Yanukovich concretó un acuerdo con Putin, en el cual logró que Rusia se comprometiera a comprar $15 \mathrm{mil} \mathrm{mi-}$ llones de dólares en bonos del Estado ucraniano y a reducir en un 33\% el precio del gas que le vende a este país, lo cual daba a Ucrania la posibilidad de aminorar su desequilibrio de balanza de pagos.

La firma de ese acuerdo radicalizó las protestas de los proeuropeos (denominados como la oposición al estar en desacuerdo con la implementación de políticas encaminadas a un mayor acercamiento a los intereses de Rusia) quienes comenzaron a exigir elecciones anticipadas, una reforma constitucional y el esclarecimiento de los términos bajo los cuales se habían aceptado los beneficios que ofreció Moscú. Posterior a ello, ocurrieron eventos que complicaron más el conflicto y agregaron un nuevo frente a las protestas, los prorrusos (o también llamados rebeldes), entre estos eventos estuvo la destitución del presidente Yanukovich -el 22 de febrero de 2014- quién fue sustituido temporalmente por Alexander Turchinov, de un perfil político más proeuropeo ${ }^{5}$ aspecto que implicó que el eje del conflicto se trasladara a Crimea (a la vez que otra ciudades eran contagiadas) que, por contar con una población en su mayoría rusohablante (76\% de sus habitantes) desconoció la legitimidad del gobierno impuesto. Lo anterior, como es sabido, resultó en la anexión de Crimea a Rusia en marzo del 2014- coadyuvada por los resultados del referéndum sobre el estatus político de Crimea y Sebastopol, que según Vladimir Putin "expresó la voluntad del pueblo crimeo" a favor de su reunificación con Rusia.

En fechas recientes ${ }^{6}$ la zona Este de Ucrania, también primordialmente rusohablante, continúa en intenso conflicto, luego de que las provincias de Donetsk y Luhansk que conforman la cuenca minera de Donbas se autoproclamaran republicas populares independientes después de celebrar referéndums similares al de Crimea. En estas provincias dominan los separatistas prorrusos, quienes piden una reforma constitucional descen-

\footnotetext{
${ }^{5}$ Quien tomó temporalmente las funciones de presidente, ante la inestabilidad política de esta nación. Relevado desde el 7 de julio de 2014 por Petro Poroshenko, actual presidente de Ucrania.

${ }^{6}$ Este documento fue escrito al cierre de 2015.
} 
tralizadora -que según un acuerdo firmado en Minsk debería suceder antes de que culmine el 2015- y dar un status especial al idioma ruso; a cambio, los dirigentes de Ucrania requieren la celebración de elecciones bajo su legislación y con la supervisión de la $\mathrm{OSCE}^{7}$ en ambas provincias, para poder concretar acuerdos con representantes legalmente elegidos. Todo esto ha mantenido a Ucrania en intenso conflicto armado y ha derivado en una perspectiva negativa en el PIB de 9\% y una inflación de cerca de $50 \%$ para finales 2015. Cabe recalcar que en el transcurso de estos acontecimientos han ocurrido diversos movimientos diplomáticos especialmente de la UE; antes del hurto de Crimea estos buscaban evitar un conflicto mayor, ahora principalmente van encaminados a resolver las diferencias entre Rusia y Ucrania en torno al gas natural.

\section{Los aspectos energéticos}

En términos generales la UE depende mucho del exterior para suplir su demanda energética, tan solo durante 2013 el 53\% de los combustibles que

\footnotetext{
${ }^{7}$ Organización para la Seguridad y la Cooperación en Europa, que fue creado con el objetivo de prevenir conflictos en Europa y sus alrededores.
}

consumió llegaron de países externos al bloque y a pesar de que ha implementado varias acciones en amparo de su seguridad energética, particularmente a raíz de la crisis del gas de 2009, la UE sigue siendo vulnerable en el plano energético, ya que su nivel de importaciones no ha podido disminuir de los 50 puntos porcentuales, al menos hasta las revisiones estadísticas más recientes; lo cual pone de manifiesto que sus acciones por disminuir la dependencia del exterior no han sido suficientes. Los dos combustibles más demandados al interior del bloque son el petróleo y el gas natural, commodities que sumados equivalen a poco más de la mitad del consumo interno de energía de la UE (véase Cuadro 1), entre los cuales el gas natural es el segundo combustible más utilizado al interior del bloque. Y aunque la UE ha disminuido su consumo de este energético especialmente desde 2010, el volumen importado contrariamente ha venido creciendo (véase Gráfica 2), lo que genera una brecha un poco incongruente -ya que el consumo viene cayendo pero las importaciones siguen elevándose- que se asocia con el desempeño mostrado por la producción de gas natural al interior del bloque, la cual descendió en un $35 \%$ en un período de 9 años, de 2004 a 2013. 


\section{Cuadro I}

UE: consumo interior bruto de energía, 2013

$-\%$ del total de energía consumida-

\begin{tabular}{|l|c|}
\hline \multicolumn{1}{|c|}{ Energético } & Porcentaje \\
\hline Petróleo & 33.4 \\
\hline Gas natural & 23.2 \\
\hline Combustibles sólidos & 17.2 \\
\hline Otros Combustibles & 26.2 \\
\hline
\end{tabular}

Nota: la suma de los porcentajes del consumo de petróleo y gas natural es de 56.6\%.

Fuente: elaboración propia con base en datos de Eurostat, 2015.
En los últimos años el bloque europeo ha obtenido este combustible principalmente de Noruega y Rusia, naciones que han logrado mantener un cierto nivel de importancia como abastecedores de dicho combustible, aventajando a otros países como Argelia que juega un papel relevante para la parte oeste del bloque. Pero quien tiene la supremacía como proveedor de gas natural frente a la UE es Rusia -que de igual modo es el segundo productor de gas a nivel mun-

\section{Gráfica 2}

Consumo de gas natural e importación de energía, 2004-2013

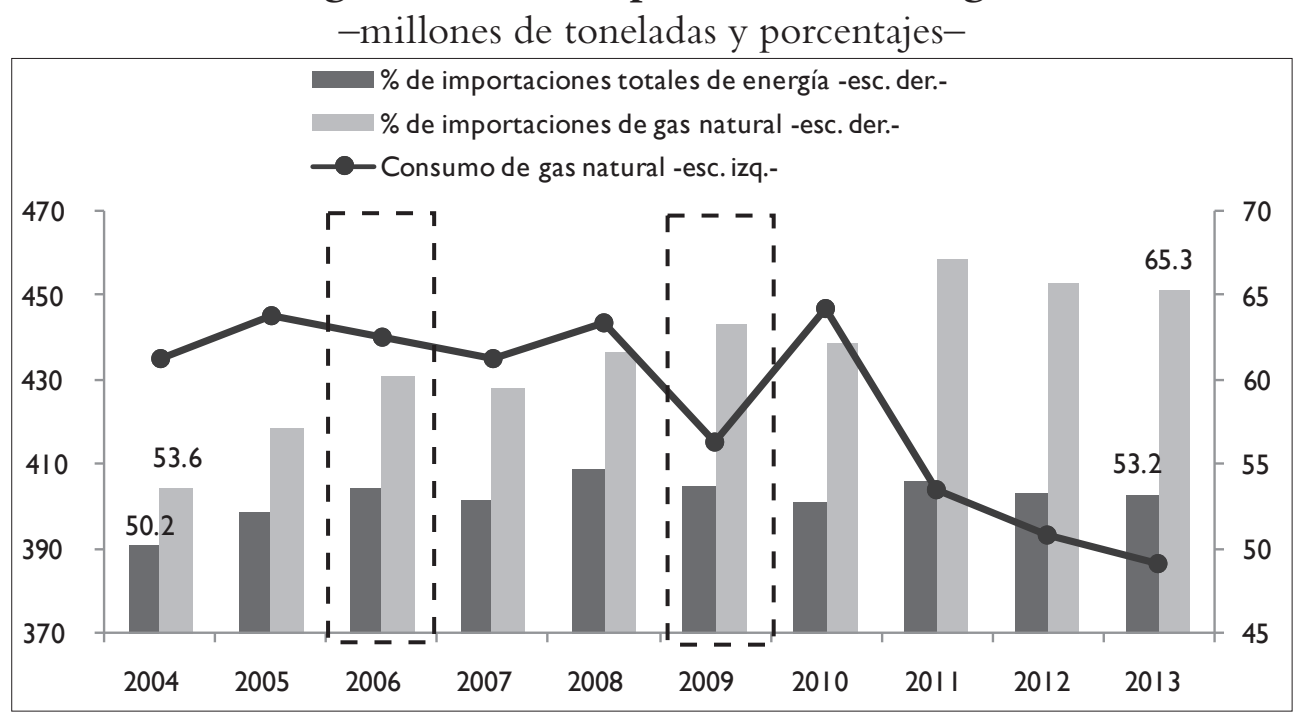

Nota: las áreas punteadas indican recortes al gas proveniente de Rusia.

Fuente: elaboración propia con base en datos de Eurostat, 2015. 


\section{Gráfica 3}

\section{UE: origen de las importaciones de gas natural, 2006-2013}

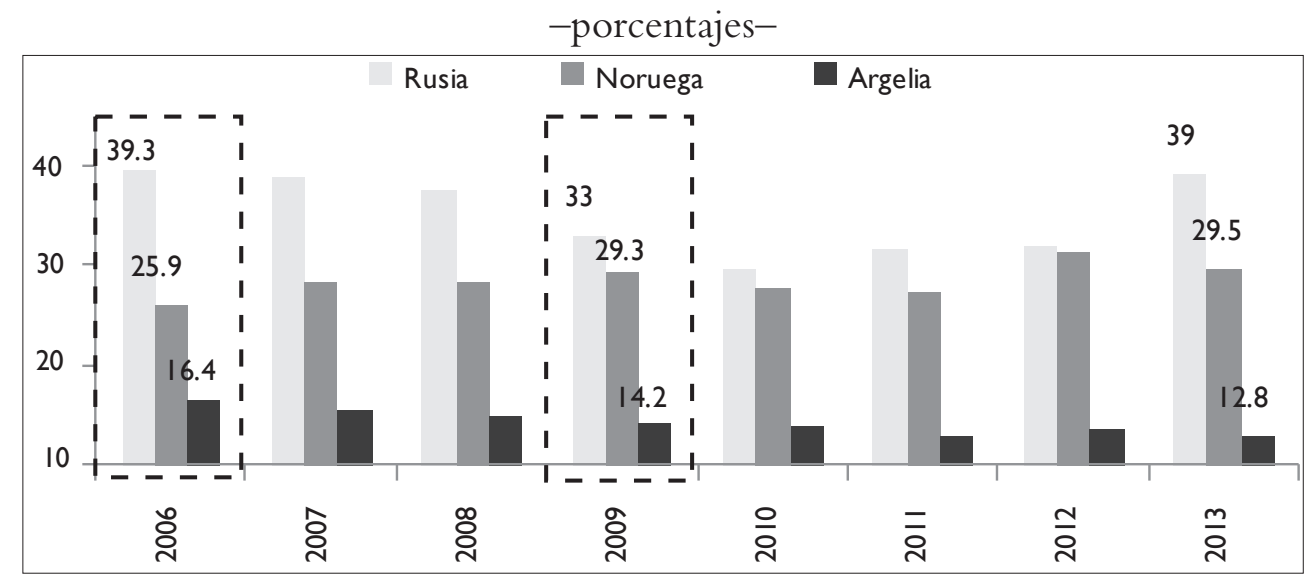

Nota: las líneas punteadas indican un recorte en el flujo de gas ruso.

Fuente: elaboración propia con base en datos de Eurostat, 2015.

dial ${ }^{8}$ - y no simplemente para este energético, también es el que más le aporta petróleo y combustibles sólidos al bloque. Dicho país cuenta con una gigante paraestatal encargada de la producción de este energético, Gazprom, la cual durante el septenio que va del 2006 a 2013 suplió en promedio $35 \%$ de las importaciones de gas natural del bloque europeo, cerrando este período con un incremento interanual de $8 \%$-diez puntos porcentuales por encima del segundo distribuidor de gas- gracias a un impulso en el nivel de importaciones que inició en 2011, ubicando a este indicador en niveles no vistos desde 2006 (véase Gráfica 3). Lo anterior es

${ }^{8}$ Segundo productor a nivel mundial, después de Estados Unidos: durante 2013 ostentó una producción de 670 bmc aproximadamente, de la cual exportó cerca de la tercera parte, 210 bmc (International Energy Agency, 2015). la muestra de que la dependencia europea al gas natural foráneo se centra en esa nación, Rusia, que se presenta como su séptimo socio comercial.

Además, al analizar la región de forma desagregada resaltan dependencias muy marcadas, pues suman nueve los países que cubren más del $75 \%$ de su consumo de gas natural únicamente con importaciones de combustible ruso, al interior de este conjunto están contenidos países como Finlandia, Republica Checa, los socios bálticos y Bulgaria naciones que llegan al extremo por ser totalmente dependientes del abasto de energía de Gazprom (véase Cuadro 2). Pero el trasfondo de la subordinación de los miembros de la UE frente a Moscú es más un problema de largo plazo, pues al interior de la 


\section{Cuadro 2}

Países europeos: importaciones de gas natural ruso, 2012

-porcentaje del total de gas natural importado-

\begin{tabular}{|l|l|l|l|l|l|l|l|l|l|}
\hline \multicolumn{2}{|c|}{$\mathbf{0 - 2 5 \%}$} & \multicolumn{2}{|c|}{$\mathbf{2 6 - 5 0 \%}$} & \multicolumn{2}{c|}{$\mathbf{5 1 - 7 5 \%}$} & \multicolumn{4}{c|}{$\mathbf{7 6 - 1 0 0 \%}$} \\
\hline Países bajos & 11.2 & Italia & 28.1 & Grecia & 59.5 & Polonia & 79.8 & Finlandia & 100 \\
\hline Francia & 15.6 & Alemania & 35.7 & Austria & 71 & Rumania & 86.1 & Estonia & 100 \\
\hline & & Eslovenia & 45.2 & & & Eslovaquia & 99.5 & Letonia & 100 \\
\hline & Hungría & 43.7 & & & Bulgaria & 100 & Lituania & 100 \\
\hline
\end{tabular}

Fuente: elaborado a partir de Financial Times abril 3, 2014.

región las interconexiones existentes para distribuir el gas son insuficientes -faltan plantas de regasificación e infraestructura de licuefacción- ya sea para irrigar el combustible al interior de la región o para acarrearlo de zonas distintas a Rusia. Otra dificultad del bloque es que sólo cuenta con 21 plantas de regasificación, ${ }^{9}$ de las cuales no hay una sola en Alemania o en Europa del Este, y por el contrario España concentra 7 de estas plantas. Es gracias a lo anterior y a que esta nación obtienen sus importaciones de gas natural de un abanico de países distintos a la parte Este de bloque, ${ }^{10}$ que España se presenta como un caso particular y escapa a la dependencia del gas natural ruso.

\footnotetext{
${ }^{9}$ Con una capacidad conjunta aproximada de 198 billones de metros cúbicos (bmc) de gas natural por año.

${ }^{10}$ A pesar de ello España no goza de autosuficiencia gasistica, de acuerdo a datos de la International Energy Agency (2015) durante 2013 esta nación contaba con una autosuficiencia energética de apenas un $0.3 \%$.
}

Bajo este tenor el actual conflicto que enfrenta Ucrania por los deseos separatistas de la cuenca minera de Donbas, es de suma importancia, ya que al interior de esta región multicultural viaja una red de gasoductos que conectan al bloque europeo con Rusia, los cuales mantienen un flujo anual de alrededor de 80 billones de metros cúbicos que corren en dirección oeste, ingresando por 7 puntos de acceso al interior de Rusia y que posterior a su paso por Ucrania desembocan en Hungría, Rumania, Eslovaquia y Polonia. Es así que Ucrania se ostenta como el corredor más grande de transporte de gas, al ser este país el tránsito obligado de la mitad de las importaciones de gas natural ruso que consume Europa (véase Cuadro 3) y que, por otro lado, para la UE la prensa manejó cifras de entre el 50 y $60 \%$ como la proporción de este combustible que transita por territorio ucraniano en dirección al bloque. 


\section{Cuadro 3}

Ucrania: terreno de traslado del gas ruso, 2010-2013

-infraestructura y billones de metros cubico-

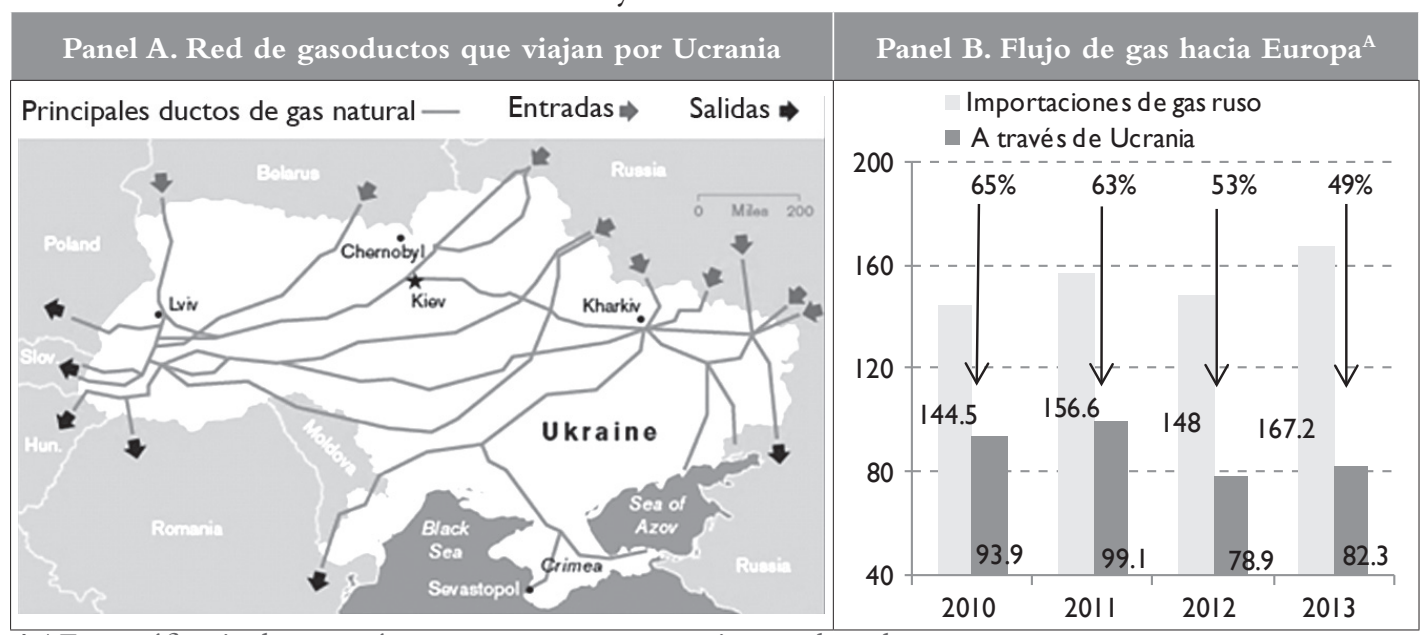

A/ Este gráfico incluye a países que no se encuentran integrados a la UE.

Fuente: elaborado con base en datos de International Energy Agency, 2014 y Bloomberg mayo 29, 2014.

Esta posición que ocupa Kiev en el paso del gas lo hace un sitio de tensión, ya que en repetidas ocasiones las discrepancias entre los empresarios de Gazprom y los gobernantes de Kiev han provocado enfrentamientos que derivaron en la suspensión del flujo de gas ruso; desde la anexión de Crimea a territorio ruso y hasta los últimos meses de 2015 se han presentado dos recortes en el suministro del gas ruso para Ucrania poniendo con ello en peligro el flujo de este combustibles hacia el bloque europeo. El primero de ellos derivó precisamente de la anexión de Crimea, ya que este evento desató un conjunto de sanciones por parte de la Estados Unidos y la UE en contra de algunos políticos y empresarios rusos por apoyar el referéndum que llevó a ello, ante esto Rusia reaccionó utilizando a la energía como un arma diplomática, aplicando un recorte en el suministro de gas que duró seis meses (de junio a diciembre de 2014), afectando a algunos países europeos (aunque no tan fuerte como se hubiera previsto). Dicho recorte se dio por causa de un desacuerdo entre Kiev y Moscú sobre el incremento del precio que aplicó Gazprom al gas natural que le vendía a Ucrania, ${ }^{11}$ so pretexto de (a) la cancelación del acuerdo de Járkov firmado en 2010, ${ }^{12}$ que quedó inva-

11 Antes de la anexión Ucrania sólo pagaba 268.5 dólares por mil metros cúbicos (d/mmc) de gas pero a partir del 1 de abril Rusia quiso aplicar una tarifa de $485.5 \mathrm{~d} / \mathrm{mmc}$ de gas.

${ }^{12}$ En virtud de este acuerdo, Ucrania extendió el arriendo de la ciudad de Sebastopol, en Cri- 
lidado después que Rusia le arrebató Crimea, y $(b)$ de la anulación del subsidio en el precio de gas que Kiev había ganado a finales de 2013 cuando Putin y Yanukovich negociaron, mismo que quedaba eliminado por la destitución de este segundo como presidente.

Aparte de lo anterior, Rusia reclamaba el pago de una deuda atrasada del gobierno ucraniano por concepto de gas por alrededor de 2500 millones de euros, y fue precisamente por la conjunción de todos estos factores que Gazprom decidió implementar en el mismo mes de la suspensión en el suministro de gas a Ucrania- un sistema de prepago que condicionó el flujo de gas al reembolso monetario oportuno de Kiev, lo cual desembocó en una serie de negociaciones tripartitas entre Rusia, la Ue y Kiev por solucionar este conflicto, pero lograron poco, solamente un acuerdo menor que permitió reanudar el flujo de gas a finales de 2014 y que no resolvía del todo el problema de la deuda ucraniana. En 2015, ya con el sistema de prepago funcionando, se presentó un nuevo recorte en el flujo de gas a causa de que Kiev no abonó su pago por adelantado, esta nueva suspensión se prolongó por 4 meses, de julio a octubre del presente año; mea, para la permanencia de la base naval rusa, con lo cual conseguía un descuento de $100 \mathrm{~d} /$ mmc de gas natural que consumía de Gazprom, manteniendo así en $400 \mathrm{~d} / \mathrm{mmc}$ el precio de este energético. tanto este recorte como el anterior fueron ampliamente divulgados por la prensa, pero estas discrepancias no son algo nuevo, ya en enero de 2009 -al igual que en 2006- Rusia interrumpió el suministro de gas a varios de sus clientes europeos - un par de semanas- por diferencias con Ucrania en torno al precio del mismo. En aquel año Moscú aplicó una disminución progresiva del bombeo de gas que pasaba por Ucrania y después que el presidente de Gazprom Alexéi Míller comprobara que éste ya no llegaba a sus socios europeos, la paraestatal decidió cerrar totalmente el flujo del mismo. ${ }^{13}$

\section{Comentarios finales}

Ucrania ha vivido peores adversidades que países como México o Estados Unidos y no ha tenido la atención que debería; en estas naciones las contracciones en el producto durante las crisis más severas no se comparan con lo que ha experimentado Ucrania. Cuando México sufrió la crisis de credibilidad en 1994, se unieron en su rescate el FMI, el Banco Mundial, el Tesoro de Estados Unidos y otros organismos más, por una caída del PIB de apenas un 6\%. Por otro lado Estados Unidos, durante la Gran

\footnotetext{
${ }^{13}$ Derivado de que el suministro con que contaba Ucrania se hacía insuficiente ante el mermado flujo de gas, esta nación comenzó a consumir el combustible que debía llegar a la UE.
} 
Depresión, tuvo una variación negativa en su producto de casi un $9 \%$, menos de la mitad que la contracción más severa en Ucrania; claro podríamos argumentar que el tamaño e importancia de esta economía y la ucraniana no tiene comparación, pero las afectaciones en términos de bienestar para la población es lo que debe pesar más en la balanza al momento de decidir los temas prioritarios.

Kiev ha mostrado caídas consecutivas en su producción de más de $10 \%$, que han ido asociadas a problemas de hiperinflación, frente a los cuales la comunidad internacional y los economistas especializados se han mostrado indiferentes; es ahora, con el problema energético -como tema de moda por las afectaciones que trae a la UE- que se ha puesto atención a lo que sucede en Ucrania. Por lo demás, los recortes al flujo de gas que viaja por territorio ucraniano, han puesto nuevamente al descubierto el problema de dependencia energética de algunos países europeos; complicación que no ha escalado a una magnitud mayor gracias a la caída en el precio de los energéticos originada por movimientos entre sus niveles de oferta y demanda mundial ante el incremento en la producción petrolera de Arabia Saudita y Estados Unidos.

Pero esta caída en el precio de la energía, que favorece a la UE, sin duda afecta a la economía ucraniana, porque esto permite que las econo- mías occidentales sigan menospreciando los problemas económicos y políticos al interior de este país. Además el problema geopolítico que vive Ucrania no se puede resolver de manera bilateral con Moscú, porque el Estado ruso tiene una concepción que lo caracteriza, y es la idea de que la absorción continua de territorios le garantiza su seguridad, lo cual deja a Ucrania en una posición de suma vulnerabilidad dado que no pertenece a la OTAN.

\section{Referencias}

Banco Mundial (2015), World Development Indicators, octubre, Washington.

Burdeina, Iuliia (2014), "Ucrania: per aspera ¿ad astra?”, Otros artificios año 1 núm. 4, octubre.

Central Intelligence Agency (2015), The world factbook-country comparison, octubre, Washington.

Chazan G. y Ed Crooks (2014), "Europe's dangerous addiction to Russian gas needs radical cure", Financial Times, 3 de abril.

Eurostat (2015), Energy statistics - quantities, annual data, septiembre-octubre, Luxemburgo.

International Energy Agency (2014), "Facts in Brief: Russia, Ukraine, Europe, Oil \& Gas - 4 March 2014”, 
Press Releases \& News 2014, 10 de marzo, París.

(2015)/ Natural gas statistics, septiembre, París.

Langley, Andrew (2014)/ "Standoff in Ukraine”, Bloomberg, 29 de mayo.

National Bank of Ukraine (2015)/ Macroeconomic Indicators (archives), octubre, Kiev.

Agencia EFE (2014-2015)/ diversas notas, varias fechas, Madrid.

BBC Mundo (2014-2015)/ diversas notas, varias fechas, Londres.

Bloomberg (2014-2015)/ diversas notas, varias fechas, Nueva York.
CNN Expansión (2014-2015)/ diversas notas, varias fechas, México.

Deutsche Welle (2014)/ diversas notas, varias fechas, Bonn, Alemania.

Diario El Economista (2013-2015)/ diversas notas, varias fechas, México.

Diario El País (2013-2015)/ diversas notas, varias fechas, Madrid.

Reuters (2014-2014)/ diversas notas, varias fechas, Nueva York.

Russia Beyond the Headlines (RBTH) (2013)/ diversas notas, varias fechas, Moscú. 